\title{
SUCCESSIVE CONVEX APPROXIMATION FOR SYSTEM PERFORMANCE OPTIMIZATION IN A MULTIUSER NETWORK WITH MULTIPLE MIMO RELAYS
}

\author{
Batu K. Chalise, Yimin D. Zhang and Moeness G. Amin \\ Center for Advanced Communications, Villanova University \\ 800 E Lancaster Avenue, PA 19085, USA \\ Email: \{batu.chalise, yimin.zhang, moeness.amin\}@villanova.edu
}

\begin{abstract}
We consider the problem of jointly optimizing amplify-andforward (AF) multiple-input multiple-output (MIMO) relays and single-antenna receivers for multipoint-to-multipoint (MP2MP) communications using weighted sum mean-squareerror (MSE) as a system performance measure. This optimization problem is nonconvex and the optimal solution cannot be obtained in polynomial time. By applying firstorder approximation for the quadratic term, we show that this problem can be approximately reformulated as a semidefinite programming (SDP) problem, which is then solved successively in an iterative way. The performance of the proposed scheme is compared with the lower bound (LB) of the global optimal solution that is obtained with increased complexity using the MSE-profile approach, and the bisection and semidefinite relaxation (SDR) techniques. Numerical results show that the performance of the proposed successive convex approximation (SCA) approach is very close to the LB of the global optimal solution and is much better than the zero-forcing MIMO relays.
\end{abstract}

Index Terms - MIMO relays, successive convex optimization, semidefinite relaxation.

\section{INTRODUCTION}

Recently, the application of multiple-input multiple-output (MIMO) relays for cooperative communications has received a significant level of interest [1]-[2]. The problem of optimizing amplify-and-forward (AF) MIMO relay is solved in [1] for a point-to-point communication case whereas in [2], the MIMO relay design is considered for the downlink of a multiuser system. In [3], semidefinite relaxation (SDR) technique is applied to solve the problem of minimizing the relay transmit power while satisfying the signalto-interference-plus-noise (SINR) requirements ( the quality

This work was supported in part by Defense Advanced Research Projects Agency (DARPA) under contract no. HR0011-10-1-0062. The views, opinions, and/or findings contained in this article/presentation are those of the author/presenter and should not be interpreted as representing the official views or policies, either expressed or implied, of the Defense Advanced Research Projects Agency or the Department of Defense. Approved for public release. Distribution unlimited. of service $(Q O S)$ approach) for all single-antenna users in a multipoint-to-multipoint (MP2MP) communication system having both perfect and imperfect instantaneous channel state information (CSI). This work is extended to multiple MIMO relays case in [4] which also solves the problem of maximizing the worst-case user SINR under individual relay power constraints (max-min approach). Although, the QoS and max-min approaches handle fairness issue in multiuser communication in an efficient manner, these methods do not necessarily optimize the overall system performance. As a result, in this work, we focus on optimizing the system performance which is characterized by weighted sum MSE. To the best of our knowledge, there has been so far no work dealing with the weighted sum MSE minimization of M2MP network. It is also worthwhile to mention that the problem of minimizing weighted sum MSE is more difficult to solve than the problems considered in [3]-[4].

In this paper, we consider the problem of minimizing the weighted sum MSE under individual relay power constraints for MP2P network that consists of multiple AF MIMO relays and single-antenna source-destination terminals. As it will be clear later in Section 4, this optimization problem can be reduced to the problem with only relay matrices. However, such problem is essentially the problem of minimizing the sum of the ratios of convex quadratic functions, which is a nonconvex optimization problem for which there exists no algorithms that can achieve global optimal solution in polynomial time [5]. In this context, instead of working with the reduced problem having only relay matrices, we deal with the original optimization problem as it is, i.e., the problem having both the relay matrices and receiver coefficients. We propose to use linear approximation of the quadratic terms of receiver coefficients that are responsible for nonconvexity of the problem. The resulting approximated convex optimization problem is reformulated as a semidefinite programming (SDP) problem, which is then solved successively in an iterative manner. We also compare the performance of the proposed scheme with the lower bound (LB) of the global optimal solution which is obtained at higher computational cost from the combined application of MSE-profile approach, and the bisection and SDR techniques. 


\section{SYSTEM MODEL}

We consider a scenario where signal transmissions from $K$ single-antenna sources to $K$ single-antenna destinations are supported by $R$ MIMO relays. Let $N_{\mathrm{r}, i}$ be the number of antennas at the $i$ th MIMO relay. It is assumed that each destination is targeted by a single source. The direct links between the sources and destinations are not considered, since we assume that these direct links undergo relatively larger path attenuations/shadow fadings compared to the links via MIMO relays. Furthermore, we assume that the MIMO relays have complete CSI, which in our case, is the knowledge of all instantaneous channels. We consider flat fading sourcerelay and relay-destination channels. The $i$ th MIMO relay processes its received signal using a linear operator $\mathbf{Z}_{i} \in$ $\mathcal{C}^{N_{\mathrm{r}, i} \times N_{\mathrm{r}, i}}$ and forwards the following signal to the destinations:

$$
\mathbf{r}_{\mathrm{o}, i}=\mathbf{Z}_{i} \sum_{k=1}^{K} \mathbf{h}_{k, i} x_{k}+\mathbf{Z}_{i} \mathbf{n}_{\mathrm{r}, i} \in \mathcal{C}^{N_{\mathrm{r}, i} \times 1}
$$

where $\mathbf{h}_{k, i} \in \mathcal{C}^{N_{\mathrm{r}, i} \times 1}$ is the channel vector between the $k$ th source and the $i$ th relay, $i \in\{1, \cdots, R\}, x_{k}$ is the signal of the $k$ th source, and $\mathbf{n}_{\mathrm{r}, i} \in \mathcal{C}^{N_{\mathrm{r}, i} \times 1}$ is the additive Gaussian noise vector at the $i$ th relay. It is assumed that $\mathrm{E}\left\{x_{k}\right\}=0$ and $\mathrm{E}\left\{\left|x_{k}\right|^{2}\right\}=1, \forall k \in\{1, K\}$. We consider $\mathbf{n}_{\mathrm{r}, i} \sim$ $\mathcal{N}_{C}\left(\mathbf{0}, \sigma_{\mathrm{r}, i}^{2} \mathbf{I}_{N_{\mathrm{r}, i}}\right)$, i.e., the entries of $\mathbf{n}_{\mathrm{r}, i}$ are zero-mean circularly symmetric complex Gaussian (ZMCSCG) with the variance $\sigma_{\mathrm{r}, i}^{2}$. The signal received by the $k$ th destination can be written as

$$
y_{\mathrm{d}, k}=\sum_{i=1}^{R} \mathbf{g}_{i, k}^{H} \mathbf{Z}_{i}\left[\sum_{j=1}^{K} \mathbf{h}_{j, i} x_{j}+\mathbf{n}_{\mathrm{r}, i}\right]+n_{\mathrm{d}, k}
$$

where $\mathbf{g}_{i, k} \in \mathcal{C}^{N_{\mathrm{r}, i} \times 1}$ contains complex conjugates of the channel coefficients from the $i$ th relay to $k$ th destination and $n_{\mathrm{d}, k}$ is the additive noise at the $k$ th destination which is assumed to be ZMCSCG, i.e., $n_{\mathrm{d}, k} \sim \mathcal{N}_{C}\left(0, \sigma_{\mathrm{d}, k}^{2}\right)$. We define

$$
\begin{aligned}
& \mathbf{a}_{k, j} \triangleq\left[\operatorname{vec}\left(\mathbf{g}_{1, k} \mathbf{h}_{j, 1}^{H}\right)^{T}, \cdots, \operatorname{vec}\left(\mathbf{g}_{R, k} \mathbf{h}_{j, R}^{H}\right)^{T}\right]^{T}, \\
& \mathbf{e}_{\mathrm{n}, k} \triangleq\left[\operatorname{vec}\left(\mathbf{g}_{1, k} \mathbf{n}_{\mathrm{r}, 1}^{H}\right)^{T}, \cdots, \operatorname{vec}\left(\mathbf{g}_{R, k} \mathbf{n}_{\mathrm{r}, R}^{H}\right)^{T}\right] \\
& \mathbf{z}_{\mathrm{L}} \triangleq\left[\mathbf{z}_{\mathrm{L}, 1}^{T}, \cdots, \mathbf{z}_{\mathrm{L}, R}^{T}\right]^{T}
\end{aligned}
$$

where $\mathbf{z}_{\mathrm{L}, i}=\operatorname{vec}\left(\mathbf{Z}_{i}\right) \in \mathcal{C}^{N_{\mathrm{r}, i}^{2} \times 1}$, and $\mathbf{a}_{k, j}$ and $\mathbf{z}_{\mathrm{L}}$ are both column vectors of length $N_{R}=\sum_{i=1}^{R} N_{\mathrm{r}, i}^{2}$. Equation (2) can be expressed as

$$
y_{\mathrm{d}, k}=\mathbf{a}_{k, k}^{H} \mathbf{z}_{\mathrm{L}} x_{k}+\sum_{j=1, j \neq k}^{K} \mathbf{a}_{k, j}^{H} \mathbf{z}_{\mathrm{L}} x_{j}+\mathbf{e}_{\mathrm{n}, k}^{H} \mathbf{z}_{\mathrm{L}}+n_{\mathrm{d}, k} .
$$

The estimated signal $\hat{x}_{k}$ at the $k$ th destination is given by $\hat{x}_{k}=w_{k} y_{\mathrm{d}, k}$ where $w_{k} \in \mathcal{C}^{1 \times 1}$ is the $k$ th receiver coefficient. The resulting $k$ th user MSE MSE $\operatorname{Me}_{k}=\mathrm{E}\left\{\left|x_{k}-\hat{x}_{k}\right|^{2}\right\}$ becomes

$$
\begin{aligned}
\operatorname{MSE}_{k}= & 1-w_{k}^{H} \mathbf{z}_{\mathrm{L}}^{H} \mathbf{a}_{k, k}-w_{k} \mathbf{a}_{k, k}^{H} \mathbf{z}_{\mathrm{L}}+ \\
& w_{k} w_{k}^{H} \mathbf{z}_{\mathrm{L}}^{H} \mathbf{R}_{\mathrm{T}, k} \mathbf{z}_{\mathrm{L}}+w_{k} w_{k}^{H} \sigma_{n_{\mathrm{d}}, k}^{2}, \text { where }
\end{aligned}
$$

$$
\mathbf{R}_{\mathrm{T}, k}=\mathbf{a}_{k, k} \mathbf{a}_{k, k}^{H}+\sum_{j=1, j \neq k}^{K} \mathbf{a}_{k, j} \mathbf{a}_{k, j}^{H}+\underbrace{\mathrm{E}_{\mathbf{n}_{\mathrm{r}, i}}\left\{\mathbf{e}_{\mathrm{n}, k} \mathbf{e}_{\mathrm{n}, k}^{H}\right\}}_{\mathbf{R}_{\mathrm{e}, k}}
$$

and $\mathbf{R}_{\mathrm{e}, k}=\operatorname{blkdiag}\left(\sigma_{\mathrm{r}, 1}^{2} \mathbf{I}_{N_{\mathrm{r}, 1}} \otimes\left[\mathbf{g}_{1, k} \mathbf{g}_{1, k}^{H}\right], \cdots, \sigma_{\mathrm{r}, R}^{2} \mathbf{I}_{N_{\mathrm{r}, R}}\right.$ $\left.\otimes\left[\mathbf{g}_{R, k} \mathbf{g}_{R, k}^{H}\right]\right)$. Note that from (3), we can write $\mathbf{z}_{\mathbf{L}, i}=$ $\mathbf{A}_{i} \mathbf{Z}_{\mathrm{L}}, \forall i$, where $\mathbf{A}_{i}=\left[\tilde{\mathbf{A}}_{i, 1}, \cdots, \tilde{\mathbf{A}}_{i, R}\right] \in \mathcal{R}^{N_{\mathrm{r}, i}^{2} \times N_{R}}, \tilde{\mathbf{A}}_{i, i}$ is an $N_{\mathrm{r}, i}^{2} \times N_{\mathrm{r}, i}^{2}$ identity matrix, and all $\left\{\tilde{\mathbf{A}}_{i, j}\right\}_{j=1, j \neq i}^{R}$ are all-zero matrices of sizes $N_{\mathrm{r}, i}^{2} \times N_{\mathrm{r}, j}^{2}$. Using (1), and noting that $\operatorname{tr}\left(\mathbf{Y}^{H} \mathbf{Y} \mathbf{X}\right)=\operatorname{vec}(\mathbf{Y})^{H}\left(\mathbf{X}^{T} \otimes \mathbf{I}_{N}\right) \operatorname{vec}(\mathbf{Y})$ for $\mathbf{X}, \mathbf{Y}$, the $i$ th relay power $P_{\mathrm{r}, i}=\mathrm{E}_{\mathbf{n}_{\mathrm{r}, i},\left\{x_{k}\right\}_{k=1}^{K}}\left\{\mathbf{r}_{\mathrm{o}, i}^{H} \mathbf{r}_{\mathrm{o}, i}\right\}$ can be written as

$$
P_{\mathrm{r}, i}=\sum_{k=1}^{K} \mathbf{z}_{\mathrm{L}}^{H} \mathbf{C}_{i, k} \mathbf{z}_{\mathrm{L}}+\sigma_{\mathrm{r}, i}^{2} \mathbf{z}_{\mathrm{L}}^{H} \mathbf{A}_{i}^{H} \mathbf{A}_{i} \mathbf{z}_{\mathrm{L}}
$$

where $\mathbf{C}_{i, k}=\mathbf{A}_{i}^{H}\left(\left(\mathbf{h}_{k, i} \mathbf{h}_{k, i}^{H}\right)^{T} \otimes \mathbf{I}_{N_{\mathrm{r}, i}}\right) \mathbf{A}_{i}$.

\section{PROPOSED JOINT OPTIMIZATION}

In this section, we present the proposed successive convex approximation (SCA) method for solving the weighted sum MSE minimization problem with the individual relay power constraints. The optimization problem can be formulated as

$$
\begin{aligned}
& \min _{\left\{w_{k}\right\}_{k=1}^{K}, \mathbf{z}_{\mathrm{L}}} f_{0} \triangleq \sum_{k=1}^{K} \beta_{k} \mathrm{MSE}_{k} \text { s. t. } \\
& \mathbf{z}_{\mathrm{L}}^{H} \mathbf{D}_{i} \mathbf{z}_{\mathrm{L}} \leq P_{i}^{\mathrm{R}}, i \in\{1, \cdots, R\}
\end{aligned}
$$

where $\mathbf{D}_{i}=\sum_{k=1}^{K} \mathbf{C}_{i, k}+\sigma_{\mathrm{r}, i}^{2} \mathbf{A}_{i}^{H} \mathbf{A}_{i},\left\{P_{i}^{\mathrm{R}}\right\}_{i=1}^{R}$ are the powers available for all relays and $\left\{\beta_{k}\right\}_{k=1}^{K} \geq 0$ are the MSE weights for all destinations. Substituting (5) into the objective function of (8), defining $\mathbf{R}_{\mathrm{I}, k} \triangleq \sum_{j=1, j \neq k}^{K} \mathbf{a}_{k, j} \mathbf{a}_{k, j}^{H}+\mathbf{R}_{\mathrm{e}, k}$, and introducing the new variables $\left\{t_{k}>0\right\}_{k=1}^{K}$ and $\tilde{w}_{k} \triangleq$ $\frac{1}{w_{k}}$, we can rewrite (8) as

$$
\begin{aligned}
& \min _{\left\{\tilde{w}_{k}, t_{k}\right\}_{k=1}^{K}, \mathbf{z}_{\mathrm{L}}} \sum_{k=1}^{K} t_{k} \text { s.t. } \\
& t_{k} \geq \beta_{k} \frac{\left|\tilde{w}_{k}-\mathbf{a}_{k, k}^{H} \mathbf{z}_{\mathrm{L}}\right|^{2}+\left(\mathbf{z}_{\mathrm{L}}^{H} \mathbf{R}_{\mathrm{I}, k} \mathbf{z}_{\mathrm{L}}+\sigma_{\mathrm{d}, k}^{2}\right)}{\left|\tilde{w}_{k}\right|^{2}}, \forall k \\
& \mathbf{z}_{\mathrm{L}}^{H} \mathbf{D}_{i} \mathbf{z}_{\mathrm{L}} \leq P_{i}^{\mathrm{R}}, \forall i .
\end{aligned}
$$

Let $\tilde{w}_{k}$ be expressed as $\tilde{w}_{k}=\operatorname{Re}\left\{\tilde{w}_{k}\right\}+j \operatorname{Im}\left\{\tilde{w}_{k}\right\}$, where $\operatorname{Re}\{\}$ and $\operatorname{Im}\{\}$ denote the real and imaginary parts of $\tilde{w}_{k}$. Then, $\left|\tilde{w}_{k}\right|^{2}$ can be written as $\left|\tilde{w}_{k}\right|^{2}=\overline{\mathbf{w}}_{k}^{T} \overline{\mathbf{w}}_{k}$, where $\overline{\mathbf{w}}_{k}^{T}=$ $\left[\operatorname{Re}\left\{\tilde{w}_{k}\right\}, \operatorname{Im}\left\{\tilde{w}_{k}\right\}\right]$. With the following definitions,

$$
\begin{aligned}
& \tilde{\mathbf{z}}_{\mathrm{L}}=\left[\mathbf{z}_{\mathrm{L}}^{T}, 1\right]^{T}, \tilde{\mathbf{R}}_{\mathrm{I}, k}=\left[\begin{array}{cc}
\mathbf{R}_{\mathrm{I}, k} & \mathbf{0}_{N_{\mathrm{R}} \times 1} \\
\mathbf{0}_{1 \times N_{\mathrm{R}}} & \sigma_{\mathrm{d}, k}^{2}
\end{array}\right] \\
& \tilde{\mathbf{D}}_{i}=\left[\begin{array}{cc}
\mathbf{D}_{i} & \mathbf{0}_{N_{\mathrm{R}} \times 1} \\
\mathbf{0}_{1 \times N_{\mathrm{R}}} & 0
\end{array}\right], \tilde{\mathbf{a}}_{k, k}=\left[\mathbf{a}_{k, k}, 0\right]^{T}(10)
\end{aligned}
$$


the optimization problem (9) can be written as

$\min _{\left\{\overline{\mathbf{w}}_{k}, t_{k}\right\}_{k=1}^{K}, \tilde{\mathbf{z}}_{\mathrm{L}}} \sum_{k=1}^{K} t_{k}$ s. t.

$\overline{\mathbf{w}}_{k}^{T} \overline{\mathbf{w}}_{k} \geq \beta_{k} \frac{\left[\overline{\mathbf{w}}_{k}-\mathbf{b}\left(\tilde{\mathbf{z}}_{\mathrm{L}}\right)\right]^{T}\left[\overline{\mathbf{w}}_{k}-\mathbf{b}\left(\tilde{\mathbf{z}}_{\mathrm{L}}\right)\right]+\tilde{\mathbf{z}}_{\mathrm{L}}^{H} \tilde{\mathbf{R}}_{\mathrm{I}, k} \tilde{\mathbf{z}}_{\mathrm{L}}}{t_{k}}, \forall k$,

$\tilde{\mathbf{z}}_{\mathrm{L}}^{H} \tilde{\mathbf{D}}_{i} \tilde{\mathbf{z}}_{\mathrm{L}} \leq P_{i}^{\mathrm{R}}, \forall i, \tilde{\mathbf{z}}_{\mathrm{L}}\left(N_{\mathrm{R}}+1\right)=1$.

where $\tilde{\mathbf{z}}_{\mathrm{L}}\left(N_{\mathrm{R}}+1\right)$ is the last element of $\tilde{\mathbf{z}}_{\mathrm{L}}$ and $\mathbf{b}\left(\tilde{\mathbf{z}}_{\mathrm{L}}\right)=$ $\left[\operatorname{Re}\left\{\tilde{\mathbf{a}}_{k, k}^{H} \tilde{\mathbf{z}}_{\mathrm{L}}\right\}, \operatorname{Im}\left\{\tilde{\mathbf{a}}_{k, k}^{H} \tilde{\mathbf{z}}_{\mathrm{L}}\right\}\right]^{T}$. In (11), define $f\left(\overline{\mathbf{w}}_{k}\right) \triangleq$ $\overline{\mathbf{w}}_{k}^{T} \overline{\mathbf{w}}_{k}$ which is convex. Thus, the first-order differentiability condition can be given as [6]

$$
f\left(\overline{\mathbf{w}}_{k}\right) \geq f\left(\overline{\mathbf{w}}_{k, 0}\right)+\left(\nabla f\left(\overline{\mathbf{w}}_{k, 0}\right)\right)^{T}\left(\overline{\mathbf{w}}_{k}-\overline{\mathbf{w}}_{k, 0}\right)
$$

for all $\overline{\mathbf{w}}_{k}$ and $\overline{\mathbf{w}}_{k, 0}$. In (12), $\nabla f\left(\overline{\mathbf{w}}_{k, 0}\right)$ is the gradient of $f\left(\overline{\mathbf{w}}_{k}\right)$ at $\overline{\mathbf{w}}_{k}=\overline{\mathbf{w}}_{k, 0}$. Since, $\nabla f\left(\overline{\mathbf{w}}_{k, 0}\right)=2 \overline{\mathbf{w}}_{k, 0}$, we can rewrite (12) as

$$
f\left(\overline{\mathbf{w}}_{k}\right) \geq 2 \overline{\mathbf{w}}_{k, 0}^{T} \overline{\mathbf{w}}_{k}-\overline{\mathbf{w}}_{k, 0}^{T} \overline{\mathbf{w}}_{k, 0} .
$$

Based on (13), the $k$ th nonconvex MSE constraint of (11), for the given feasible $\overline{\mathbf{w}}_{k, 0}$, can be written as

$$
\begin{aligned}
& 2 \overline{\mathbf{w}}_{k, 0}^{T} \overline{\mathbf{w}}_{k}-\overline{\mathbf{w}}_{k, 0}^{T} \overline{\mathbf{w}}_{k, 0}-\beta_{k} \tilde{\mathbf{x}}^{T} t_{k}^{-1} \tilde{\mathbf{x}} \\
& -\beta_{k} \tilde{\mathbf{z}}_{\mathrm{L}}^{H} \tilde{\mathbf{R}}_{\mathrm{I}, k} t_{k}^{-1} \tilde{\mathbf{z}}_{\mathrm{L}} \geq 0
\end{aligned}
$$

where $\tilde{\mathbf{x}} \triangleq\left[\overline{\mathbf{w}}_{k}-\mathbf{b}\left(\tilde{\mathbf{z}}_{\mathrm{L}}\right)\right]$. Applying Schur-complement theorem [6] in (14), we can write (14) as

$\left[\begin{array}{cc}t_{k} \mathbf{I}_{2} & \sqrt{\beta_{k}} \tilde{\mathbf{x}} \\ \sqrt{\beta_{k}} \tilde{\mathbf{x}}^{T} & 2 \overline{\mathbf{w}}_{k, 0}^{T} \overline{\mathbf{w}}_{k}-\overline{\mathbf{w}}_{k, 0}^{T} \overline{\mathbf{w}}_{k, 0}-\beta_{k} \tilde{\mathbf{z}}_{\mathrm{L}}^{H} \tilde{\mathbf{R}}_{\mathrm{I}, k} t_{k}^{-1} \tilde{\mathbf{z}}_{\mathrm{L}}\end{array}\right] \succeq 0$ which can be re-expressed as

$$
\overline{\mathbf{A}}_{k}-t_{k}^{-1} \mathbf{B}_{k} \succeq 0
$$

where

$$
\begin{aligned}
& \overline{\mathbf{A}}_{k}=\left[\begin{array}{cc}
t_{k} \mathbf{I}_{2} & \sqrt{\beta_{k}} \tilde{\mathbf{x}} \\
\sqrt{\beta_{k}} \tilde{\mathbf{x}}^{T} & 2 \overline{\mathbf{w}}_{k, 0}^{T} \overline{\mathbf{w}}_{k}-\overline{\mathbf{w}}_{k, 0}^{T} \overline{\mathbf{w}}_{k, 0}
\end{array}\right] \\
& \mathbf{B}_{k}=\left[\begin{array}{cc}
\mathbf{0}_{2 \times 2} & \mathbf{0}_{2 \times 1} \\
\mathbf{0}_{1 \times 2} & \sqrt{\beta_{k}} \tilde{\mathbf{z}}_{\mathrm{L}}^{H} \tilde{\mathbf{R}}_{\mathrm{I}, k}^{\frac{1}{2}} \tilde{\mathbf{R}}_{\mathrm{I}, k}^{\frac{1}{2}} \tilde{\mathbf{z}}_{\mathrm{L}} \sqrt{\beta_{k}}
\end{array}\right] .
\end{aligned}
$$

Note that $\mathbf{B}_{k}$ can be decomposed as $\mathbf{B}_{k}=\tilde{\mathbf{B}}_{k}^{H} \tilde{\mathbf{B}}_{k}$, where

$$
\tilde{\mathbf{B}}_{k}=\left[\mathbf{0}_{\left(N_{\mathrm{R}}+1\right) \times 2}, \sqrt{\beta_{k}} \tilde{\mathbf{R}}_{\mathrm{I}, k}^{\frac{1}{2}} \tilde{\mathbf{z}}_{\mathrm{L}}\right] .
$$

Using Schur-complement theorem in (15), we have

$$
\left[\begin{array}{cc}
t_{k} \mathbf{I}_{N_{\mathrm{R}}+1} & \tilde{\mathbf{B}}_{k} \\
\tilde{\mathbf{B}}_{k}^{H} & \overline{\mathbf{A}}_{k}
\end{array}\right] \succeq 0 .
$$

From (15)-(18), we obtain

$\min _{\left\{\overline{\mathbf{w}}_{k}, t_{k}\right\}_{k=1}^{K}, \tilde{\mathbf{z}}_{\mathrm{L}}} \sum_{k=1}^{K} t_{k}$ s. t.

$\left[\begin{array}{ccc}t_{k} \mathbf{I}_{N_{\mathrm{R}}+1} & \mathbf{0}_{\left(N_{\mathrm{R}}+1\right) \times 2} & \sqrt{\beta_{k}} \tilde{\mathbf{R}}_{\mathrm{I}, k}^{\frac{1}{2}} \tilde{\mathbf{z}}_{\mathrm{L}} \\ \mathbf{0}_{2 \times\left(N_{\mathrm{R}}+1\right)} & t_{k} \mathbf{I}_{2} & \sqrt{\beta_{k}} \tilde{\mathbf{x}} \\ \sqrt{\beta_{k}} \tilde{\mathbf{z}}_{\mathrm{L}}^{H} \tilde{\mathbf{R}}_{\mathrm{I}, k}^{\frac{1}{2}} & \sqrt{\beta_{k}} \tilde{\mathbf{x}}^{T} & 2 \overline{\mathbf{w}}_{k, 0}^{T} \overline{\mathbf{w}}_{k}-\overline{\mathbf{w}}_{k, 0}^{T} \overline{\mathbf{w}}_{k, 0}\end{array}\right] \succeq 0$,

$\forall k,\left\|\tilde{\mathbf{z}}_{\mathrm{L}}^{H} \tilde{\mathbf{D}}_{i}^{\frac{1}{2}}\right\| \leq \sqrt{P_{i}^{\mathrm{R}}}, \forall i, \tilde{\mathbf{z}}_{\mathrm{L}}\left(N_{\mathrm{R}}+1\right)=1$.
The above SDP formulation is an approximation to problem (11) at some feasible solution $\overline{\mathbf{w}}_{k, 0}$. The quality of this approximation can be improved by taking the solution $\overline{\mathbf{w}}_{k}$ of current iteration as the feasible solution for the next iteration. This results into our proposed SCA algorithm which is summarized in Table I. In each iterative step, the convex problem

\section{TABLE I - SCA Algorithm :}

Step 1: Initialize feasible $\overline{\mathbf{w}}_{k, 0}$ and set the maximum number of iterations $l_{\max }$.

Step 2: Solve the convex optimization problem (19) to obtain $\left\{\overline{\mathbf{w}}_{k}^{l}\right\}_{k=1}^{K}, \tilde{\mathbf{z}}_{\mathrm{L}}^{l}$, and $\left\{t_{k}^{l}\right\}_{k=1}^{K}$.

Step 3: Update $\overline{\mathbf{w}}_{k, 0}$ as $\overline{\mathbf{w}}_{k, 0}^{l+1}=\overline{\mathbf{w}}_{k}^{l}, \forall k$.

Step 4: Go to step 2 until $\left|f_{0}^{l+1}-f_{0}^{l}\right| \leq \epsilon_{1}$ or $l=l_{\text {max }}$.

(19) is solved with the improved feasible solution $\overline{\mathbf{w}}_{k, 0}$. Thus, at each iteration of the SCA Algorithm, the objective function decreases. Furthermore, since the sum MSE objective function is lower bounded by 0 , the algorithm is guaranteed to converge. However, convergence to global optimality cannot be guaranteed. More details on convergence and optimality of this algorithm are left for the journal version of this paper.

\section{LOWER BOUND VIA SDR APPROACH}

Since the relay power constraints do not depend on $\left\{w_{k}\right\}_{k=1}^{K}$, the optimal $\left\{w_{k}\right\}_{k=1}^{K}$ for the given $\mathbf{z}_{\mathrm{L}}$ can be obtained by solving the first-order derivative of (5) w.r.t. $w_{k}$. This results into the following minimum MSE solution:

$$
w_{k}=\frac{\mathbf{z}_{\mathrm{L}}^{H} \mathbf{a}_{k, k}}{\mathbf{z}_{\mathrm{L}}^{H} \mathbf{R}_{\mathrm{T}, k} \mathbf{z}_{\mathrm{L}}+\sigma_{n_{\mathrm{d}}, k}^{2}}, \forall k .
$$

Substituting (20) into (5), we can obtain the weighted sum MSE as a function of $\mathbf{z}_{\mathrm{L}}$. This step along with some simple derivations, yields the following weighted sum MSE:

$$
\tilde{f}_{0}=\sum_{k=1}^{K} \beta_{k} \underbrace{\left[\frac{\mathbf{z}_{\mathrm{L}}^{H} \mathbf{R}_{\mathrm{I}, k} \mathbf{z}_{\mathrm{L}}+\sigma_{n_{\mathrm{d}}, k}^{2}}{\mathbf{z}_{\mathrm{L}}^{H} \mathbf{R}_{\mathrm{T}, k} \mathbf{z}_{\mathrm{L}}+\sigma_{n_{\mathrm{d}}, k}^{2}}\right]}_{\operatorname{MSE}_{k}\left(\mathbf{z}_{\mathrm{L}}\right)}
$$

which is nonconvex function of $\mathbf{z}_{\mathrm{L}}$. Hence, the following power constrained weighted sum MSE minimization problem

$$
\min _{\mathbf{z}_{\mathrm{L}}} \tilde{f}_{0} \text { s.t. } \mathbf{z}_{\mathrm{L}}^{H} \mathbf{D}_{i} \mathbf{z}_{\mathrm{L}} \leq P_{i}^{\mathrm{R}}, \forall i
$$

is nonconvex which cannot be solved globally in polynomial time. We know that $\operatorname{MSE}_{k}\left(\mathbf{z}_{\mathrm{L}}\right)$ can be expressed as $\operatorname{MSE}_{k}\left(\mathbf{z}_{\mathrm{L}}\right)=\gamma_{k} \tilde{f}_{0}, \forall k$ where $\left\{0 \leq \gamma_{k} \leq 1\right\}_{k=1}^{K}$ and $\sum_{k=1}^{K} \gamma_{k}=1$. This means that $\gamma_{k}$ quantifies $k$ th user's contribution to the overall MSE. For a given $\gamma=\left[\gamma_{1}, \gamma_{2}, \cdots, \gamma_{K}\right]$, which we call MSE-profile vector in analogy to the rateprofile concept in [7], we can rewrite (22) as

$$
\begin{aligned}
& \min _{\mathbf{z}_{\mathrm{L}}, \tilde{f}_{0}} \tilde{f}_{0} \text { s.t. } \operatorname{MSE}_{k}\left(\mathbf{z}_{\mathrm{L}}\right) \leq \gamma_{k} \tilde{f}_{0}, \forall k, \\
& \mathbf{z}_{\mathrm{L}}^{H} \mathbf{D}_{i} \mathbf{z}_{\mathrm{L}} \leq P_{i}^{\mathrm{R}}, \forall i .
\end{aligned}
$$


Using $\mathrm{MSE}_{k}$ from (21) in (23), defining $\tilde{\mathbf{Z}} \triangleq \mathbf{z}_{\mathrm{L}} \mathbf{z}_{\mathrm{L}}^{H}$ and relaxing the rank-one constraint of $\tilde{\mathbf{Z}}$, we can write (23) as

$$
\begin{aligned}
& \min _{\tilde{\mathbf{Z}}, \tilde{f}_{0}} \tilde{f}_{0} \text { s.t. } \\
& \operatorname{tr}\left(\left(\gamma_{k} \tilde{f}_{0} \mathbf{R}_{\mathrm{T}, k}-\mathbf{R}_{\mathrm{I}, k}\right) \tilde{\mathbf{Z}}\right)+\left(\gamma_{k} \tilde{f}_{0}-1\right) \sigma_{n_{\mathrm{d}}, k}^{2} \geq 0, \forall k \\
& \operatorname{tr}\left(\tilde{\mathbf{Z}} \mathbf{D}_{i}\right) \leq P_{i}^{\mathrm{R}}, \forall i .
\end{aligned}
$$

For the given profile vector $\gamma$ and $\tilde{f}_{0}$, this is a quasiconvex function w.r.t. $\tilde{\mathbf{Z}}$ which can be solved using bisection search [4], [8]. At each step of the bisection search over $\tilde{f}_{0}$, we solve the SDR feasibility problem for (24) w.r.t. $\tilde{\mathbf{Z}}$ at $\tilde{f}_{\mathrm{m}}=\frac{\tilde{f}_{1}+\tilde{f}_{\mathrm{u}}}{2}$ where $\tilde{f}_{1}$ and $\tilde{f}_{\mathrm{u}}$ are, respectively, the lower and upper bounds for $\tilde{f}_{0}$. If the problem is feasible, we set $\tilde{f}_{\mathrm{u}}=\tilde{f}_{\mathrm{m}}$, otherwise we set $\tilde{f}_{1}=\tilde{f}_{\mathrm{m}}$. Now, it remains to apply this process for all possible $\gamma$. For this purpose, we first divide the interval $[0,1]$ of each $\gamma_{k}$ into $L$ equal divisions to get $\gamma_{k, n}=\frac{n}{L}$, $n=0,1, \cdots L$. Then, from $(L+1)^{K}$ values of $\gamma_{k, n}$, we select the combinations of $\gamma_{k, n}$ for which $\sum_{k=1}^{K} \gamma_{k, n}=1$. The selected combinations form all possible profile vectors $\gamma$. The relaxed problem (24) is then solved for all aforementioned $\gamma$ and the $\tilde{\mathbf{Z}}$ that gives the best (minimum) $\tilde{f}_{0}$ is taken as the optimal solution. However, the computational complexity of this approach increases rapidly with $K$ and $N$. Moreover, since the rank-one constraint in (24) is relaxed, in general, we will only obtain LB to the global optimal solution of (22). Nevertheless, this bound serves as an important benchmark for assessing the performance of the proposed SCA approach.

\section{NUMERICAL RESULTS AND DISCUSSIONS}

For numerical simulations, we take $N_{\mathrm{r}, i}=N_{\mathrm{r}}$ and $\sigma_{\mathrm{r}, i}^{2}=\sigma_{\mathrm{r}}^{2}$ for all $i=1, \cdot \cdot R, \sigma_{\mathrm{d}, k}^{2}=\sigma_{\mathrm{d}}^{2}$ and $\beta_{k}=1$ for all $k=1, \cdot \cdot K$, $K=3, R=2$, and $\epsilon_{1}=10^{-4}$. All channel coefficients are zero-mean complex Gaussian distributed with the unit variance. For comparison, we also include the performance of zero-forcing (ZF) MIMO relays (applicable for $N_{\mathrm{r}} \geq K$ ) for the case $N_{\mathrm{r}}=K$. These ZF MIMO relays are given by $\mathbf{Z}_{i}=\alpha_{i} \mathbf{G}_{i}\left[\mathbf{G}_{i}^{H} \mathbf{G}_{i}\right]^{-1}\left[\mathbf{H}_{i}^{H} \mathbf{H}_{i}\right]^{-1} \mathbf{H}_{i}^{H}, \forall i$, where $\mathbf{G}_{i}=$ $\left[\mathbf{g}_{i, 1}, \cdot \cdot \mathbf{g}_{i, K}\right]$ and $\mathbf{H}_{i}=\left[\mathbf{h}_{1, i}, \cdots \mathbf{h}_{K, i}\right]$ are $N_{\mathrm{r}} \times K$ matrices and $\alpha_{i}$ is the scaling factor that needs to be properly selected for satisfying the power constraint of $i$ th MIMO relay. The linear receivers for the latter case are taken as $\left\{w_{k}\right\}_{k=1}^{K}=1$.

All simulation results are obtained by averaging over 100 independent channel realizations. The convex problem (19) and the convex feasibility problem in (24) are solved using the CVX software. The convergence precision for bisection search in (24) is set to 0.001. In Figure 1(a), we compare the average sum MSE as a function of $\sigma_{\mathrm{d}}^{2}$, obtained from the proposed SCA with the LB of the global optimal solution. In this figure, we take $\sigma_{\mathrm{r}}^{2}=-15 \mathrm{~dB}$ and $P_{i}^{\mathrm{R}}=3 \mathrm{~dB}$ for all $i$. As expected, in all methods of Figure 1(a), the sum MSE improves when $\sigma_{\mathrm{d}}^{2}$ decreases. Furthermore, it can be observed from this

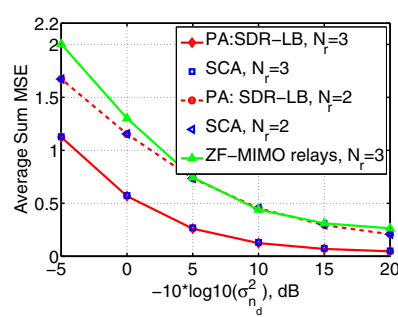

(a) Sum MSE versus $\sigma_{d}^{2}$

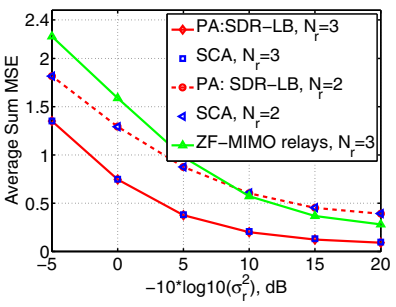

(b) Sum MSE versus $\sigma_{\mathrm{r}}^{2}$
Fig. 1. Performance of the proposed SCA and LB to the global optimal.

figure that there exists negligible difference between the proposed method and the LB to the global optimal solution. For $N_{\mathrm{r}}=3$, it can also be seen from Figure 1(a) that the proposed SCA method significantly outperforms the case with ZF MIMO relays. The average sum MSE versus $\sigma_{\mathrm{r}}^{2}$ is displayed in Figure 1(b) for the proposed method with $\sigma_{\mathrm{d}}^{2}=-10$ $\mathrm{dB}$. Again as a reference, the performance of the ZF MIMO relays is shown for $N_{\mathrm{r}}=3$. It can be observed from Figure 1(b) that the proposed approach is very close to the LB of the global solution. Moreover, in contrast to the computationally prohibitive grid search required to obtain the LB solution, the SCA scheme converges in few iterations, e.g., in average, only 10 iterations are required with the initialization $\overline{\mathbf{w}}_{k, 0}=[1,0]^{T}, \forall k, N_{\mathrm{r}}=2$ and $\sigma_{\mathrm{r}}^{2}=\sigma_{\mathrm{d}}^{2}=-15 \mathrm{~dB}$.

\section{REFERENCES}

[1] O. Munoz, J. Vidal, and A. Augustin, "Linear transceiver design in nonregenerative relays with channel state information," IEEE Trans. Sig. Proc., vol. 55, pp. 2593-2604, Jun. 2007.

[2] C. Chae, T. Tang, R. W. Heath Jr., and S. Cho, "MIMO relaying with linear processing for multiuser transmission in fixed relay networks," IEEE Trans. Sig. Proc., vol. 56, no. 2, pp. 727-738, Feb. 2008.

[3] B. K. Chalise and L. Vandendorpe, "MIMO relay design for multipoint-to-multipoint communications with imperfect channel state information," IEEE Trans. Sig. Proc., vol. 57, no. 7, pp. 2785-2796, July 2009.

[4] —-, "Optimization of MIMO relays for multipoint-tomultipoint communications: Nonrobust and robust designs," IEEE Trans. Sig. Proc., vol. 58, no. 12, pp. 6355-6368, Dec. 2010.

[5] P.-P. Shen, Y.-P. Duan, Y.-G. Pei, "A simplicial branch and duality bound algorithm for the sum of convexconvex ratios problem," J. Comput. Appl. Math., vol. 223, pp. 145-158, 2009.

[6] S. Boyd and L. Vandenberghe, Convex optimization, Cambridge University Press, 2004.

[7] R. Zhang and S. Cui, "Cooperative interference management with MISO beamforming," IEEE Trans. Sig. Proc., vol. 58, no. 10, pp. 5450-5458, Oct. 2010.

[8] E. Karipidis, N. D. Sidiropoulos, and Z-Q. Luo, "Quality of service and max-min fair transmit beamforming to multiple cochannel multicast groups," IEEE Trans. Sig. Proc., vol. 56, no 3, pp. 1268-1279, Mar. 2008. 